\title{
Parental Acceptance of a Mandatory Human Papillomavirus (HPV) Vaccination Program
}

\author{
Daron Ferris, MD, Leslie Horn, BS, and Jennifer L. Waller, PhD
}

Objectives: The objective of this study was to determine factors that influence parent's acceptance of a mandatory school-based human papillomavirus (HPV) vaccination program.

Methods: A convenience sample of 325 parents, with children aged 9 to 17 years old, completed a 53-item survey. Survey questions targeted their opinions about HPV, the HPV vaccine, and a mandatory HPV vaccination program. $\chi^{2}$ tests were used to examine relationships between survey items.

Results: Characteristics of parents who believed the HPV vaccine should be mandated included limited financial resources $(P=.03)$, history of HPV-related disease $(P=.04)$, understanding their child's susceptibility $(P=.03)$, interest in HPV vaccination for their child $(P=.0001)$, and knowledge that the vaccine reduces the risk of cervical cancer $(P=.001)$. Parents of children aged 12 to 14 years old $(P=$ $.02)$ or who knew the vaccine reduced their child's risk of developing genital warts $(P=.02)$ and cervical cancer $(P=.001)$ would be more likely to comply with a mandatory HPV vaccine program.

Conclusions: Certain characteristics define parents who support a mandatory HPV vaccination program. Greater education of parents and health care providers should improve vaccination uptake, which ultimately reduces morbidity and mortality from HPV related diseases. (J Am Board Fam Med 2010;23: 220-229.)

Keywords: Human Papillomavirus, Health Care Surveys, Attitude, Vaccination

Although the quadrivalent human papillomavirus (HPV) vaccine has demonstrated robust efficacy and reasonable safety, suboptimal vaccination rates raise concern and questions. ${ }^{1-6}$ A survey conducted in 2007 by the Centers for Disease Control and Prevention found that approximately $25 \%$ of 13 - to 17-year-old girls had received at least one dose of the HPV vaccine. ${ }^{5}$ Moreover, far fewer 9- to 12 -

This article was externally peer reviewed.

Submitted 22 April 2009; revised 8 July 2009; accepted 13 July 2009.

From the Gynecologic Cancer Prevention Center (DF); the Departments of Family Medicine and Obstetrics and Gynecology (DF) and the Department of Biostatistics (JLW), Medical College of Georgia (LH), Augusta, Georgia.

Funding: Residual grant funds.

Conflict of interest: Dr. Ferris is an investigator for GlaxoSmithKline vaccine studies. He is also a consultant, investigator, and speaker for Merck and Co., Inc. Ms. Horn and Dr. Waller have no potential conflicts.

Corresponding author: Dr. Daron G. Ferris, Department of Family Medicine, Medical College of Georgia, 1423 Harper Street, HH-105, Augusta, GA 30912 (E-mail: agerman@ mcg.edu).

\footnotetext{
See Related Commentary on Page 149.
}

year-old and 18- to 26-year-old girls and women have initiated the vaccination series. The poor rate of HPV vaccine coverage for 9- to 12-year-old girls is particularly worrisome because this has been deemed the ideal age to vaccinate. ${ }^{7}$ In comparison, coverage for school-entry vaccines during the 2007-2008 school year estimated by the Centers for Disease Control and Prevention varied between 94\% (varicella) and 96\% (hepatitis B). ${ }^{8}$ The differences in these vastly contrasting rates of vaccination are likely because of many factors, including cost, safety, knowledge, provider issues, access, and controversy. ${ }^{9-11}$ However, one simple explanation is that school-entry vaccines are mandated and the $\mathrm{HPV}$ vaccine is not.

Although discussion of mandating vaccines generates conflicting opinions, this approach has proven very beneficial from a public health perspective. School-mandated vaccination programs have increased coverage for other vaccines. ${ }^{12-15}$ However, such an approach for the HPV vaccine has met some resistance by the medical community. ${ }^{16}$ Further, only a minority of parents approve mandating the quadrivalent $\mathrm{HPV}$ vaccine. ${ }^{17,18}$ Loss of parental autonomy, cost, insufficient data from clinical trials, and too 
many side effects are the main reasons for lack of parental support. ${ }^{17,18}$ In addition, critical appraisal of parental acceptance of a mandatory HPV vaccination program would be beneficial before promoting required HPV vaccination for children. The purpose of this study was to determine factors that influence parental acceptance of a mandatory $H P V$ vaccination program.

\section{Materials and Methods}

We asked parents of 9- to 17-year-old children to participate in this study. A convenience sample of eligible parents was recruited from waiting rooms in family medicine, obstetrics and gynecology, and pediatric clinics at the Medical College of Georgia, Augusta, and from Women's Health Associates, Atlanta, Georgia. Patients were also recruited from community sites, including Riverview Park, North Augusta, South Carolina; Suwanee Academy of the Arts, Suwanee, Georgia; and hair and nail salons in Augusta and Atlanta. We excluded parents who were unable to read English. This study was approved by the Human Assurance Committee at the Medical College of Georgia.

The questionnaire has been described previously, but it consisted of 53 questions that collected demographic data, opinions about HPV and vaccines, attitudes about the HPV vaccine, and issues involving a mandatory HPV vaccination program. ${ }^{17}$ The main outcome questions determined parental reactions to a mandated HPV vaccination program. Specifically, these questions were (1) Do you think the HPV vaccine should be added to the list of school entry vaccine requirements? and (2) If the vaccine was mandatory for a child to attend school, which of the following statements most agrees with your view? (answer options for question 2 were "I would sign a waiver to prevent my child from receiving the vaccine"; "I would have my child vaccinated"; or "I would do whatever my doctor recommends." Questions were structured as yes/no and multiple choice. Some pertinent questions from previously developed questionnaires were included. ${ }^{19-21}$

Parents were asked to participate in a study about their opinions of the HPV vaccine and mandatory vaccination programs. Eligible parents who agreed to participate were given a 1-page cover letter that described HPV and the HPV vaccine. Subjects then completed the self-administered, anonymous survey and returned it in an envelope to the investigators.

Descriptive statistics, including means and standard deviations, frequencies, and percentages, were calculated for responses to each question. $\chi^{2}$ tests were used to examine the relationship between various questionnaire items. All statistical analyses were performed using SAS software version 9.1.3 (SAS Institute, Inc., Cary, NC) and statistical significance was assessed using an $\alpha$ level of 0.05 .

\section{Results}

We asked 365 parents to participate in the study; 325 parents enrolled in the fall of 2008. The response rate was $89 \%$, with insufficient time and length of the questionnaire being the main reasons for parents' refusal to participate. Complete demographic data based on the 325 parents have been published previously. ${ }^{17}$ In summary, subjects tended to be female, 30 to 39 years old, white, protestant, have a family income between $\$ 20,000$ and $\$ 50,000$, have some college education, and have private health insurance. Personal history of HPV-related problems included $13 \%$ with a previous HPV infection, 5\% with condyloma, and 37\% had a previous abnormal Papanicolaou test result.

Associations between demographic variables and agreement with adding the HPV vaccine to school entry requirements are seen in Table 1 . Those with incomes $<\$ 20,000$, those with no health insurance, Medicaid, or Tricare insurance, or those who have ever had HPV were more likely to agree that the HPV vaccine should be added to school entry requirements. Sex, race, and age of children did not predict support for an HPV vaccine mandate.

Associations between various other variables and agreement with mandating the HPV vaccine were also examined (Table 2). Subjects who were not familiar or very familiar with HPV felt it very likely that their child could contract HPV and thought it was very important for their child to receive the HPV vaccine; those who thought that the HPV vaccine reduced the risk of cervical cancer more frequently thought that the HPV vaccine should be added to school entry requirements. Parents who supported government-mandated vaccines thought a mandatory HPV vaccine program would be very helpful, and those who want the federal government to help pay for the HPV vaccine more often supported school entry requirements. 
Table 1. Association between Demographic Variables and Parent's Agreement with Adding the Human Papilloma Virus Vaccine to School Entry Requirements

\begin{tabular}{|c|c|c|c|}
\hline \multirow[b]{2}{*}{ Variable } & \multicolumn{2}{|c|}{$\begin{array}{l}\text { Do you Think the HPV Vaccine should be } \\
\text { Added to the List of School Entry Vaccine } \\
\text { Requirements? }\end{array}$} & \multirow[b]{2}{*}{$P^{*}$} \\
\hline & No (n [\%]) & Yes (n [\%]) & \\
\hline \multicolumn{3}{|l|}{ Gender } & .47 \\
\hline Male & $19(51.4)$ & $18(48.7)$ & \\
\hline Female & $163(57.6)$ & $120(42.4)$ & \\
\hline $18-29$ & $30(53.6)$ & $26(46.4)$ & \\
\hline $30-39$ & $81(55.9)$ & $64(44.1)$ & \\
\hline $40-49$ & $49(54.4)$ & $41(45.6)$ & \\
\hline $50+$ & $22(75.9)$ & $7(24.1)$ & \\
\hline \multicolumn{3}{|l|}{ Race } & .50 \\
\hline White & $103(56.6)$ & $79(43.4)$ & \\
\hline \multicolumn{3}{|l|}{ Ages of Children, 9-11 } & .91 \\
\hline No & $75(57.3)$ & $56(42.8)$ & \\
\hline Yes & $107(56.6)$ & $82(43.4)$ & \\
\hline \multicolumn{3}{|l|}{ Ages of Children, 12-14 } & .05 \\
\hline No & $126(60.9)$ & $81(39.1)$ & \\
\hline Yes & $56(49.6)$ & $57(50.4)$ & \\
\hline \multicolumn{3}{|l|}{ Ages of Children, 15-17 } & .80 \\
\hline No & $112(57.4)$ & $83(42.6)$ & \\
\hline Yes & $70(56.0)$ & $55(44.0)$ & \\
\hline \multicolumn{3}{|l|}{ Income } & .03 \\
\hline \multicolumn{3}{|l|}{ Health insurance } & .01 \\
\hline None & $9(50.0)$ & $9(50.0)$ & \\
\hline Private & $135(61.6)$ & $84(38.4)$ & \\
\hline Medicaid & $26(40.6)$ & $38(59.4)$ & \\
\hline Medicare & $2(100.0)$ & $0(0.0)$ & \\
\hline Other & $9(75.0)$ & $3(25.0)$ & \\
\hline Tricare & $1(20.0)$ & $4(80.0)$ & \\
\hline \multicolumn{3}{|l|}{ Ever had HPV } & .04 \\
\hline Yes & $18(42.9)$ & $24(57.1)$ & \\
\hline No & $155(60.3)$ & $102(39.7)$ & \\
\hline Not sure & $9(42.9)$ & $12(57.1)$ & \\
\hline \multicolumn{3}{|l|}{ Ever had genital warts } & .78 \\
\hline Yes & $8(53.3)$ & $7(46.7)$ & \\
\hline No & $174(57.1)$ & $131(43.0)$ & \\
\hline \multicolumn{3}{|l|}{ Abnormal Papanicolaou test (women only) } & .55 \\
\hline Yes & $67(56.8)$ & $51(43.2)$ & \\
\hline No & $98(58.7)$ & $69(41.3)$ & \\
\hline Not sure & $17(48.6)$ & $18(51.4)$ & \\
\hline \multicolumn{4}{|c|}{$\begin{array}{l}{ }^{*} \chi^{2} \text { test. } \\
\text { HPV, human papillomavirus; GED, General Educational Development test. }\end{array}$} \\
\hline
\end{tabular}


Table 2. Association of Parental Attitudes, Beliefs, and Agreement with Adding the Human Papillomavirus Vaccine to School Entry Requirements

\begin{tabular}{|c|c|c|c|}
\hline \multirow[b]{2}{*}{ Variable } & \multicolumn{2}{|c|}{$\begin{array}{l}\text { Do You Think the HPV Vaccine } \\
\text { Should be Added to the List of School } \\
\text { Entry Vaccine Requirement? }\end{array}$} & \multirow[b]{2}{*}{$P^{*}$} \\
\hline & No (n [\%]) & Yes (n [\%]) & \\
\hline Support government-mandated vaccine requirements & & & $<.0001$ \\
\hline No & $33(97.1)$ & $1(2.9)$ & \\
\hline Yes & $148(51.9)$ & $137(48.1)$ & \\
\hline Familiar with HPV & & & .02 \\
\hline Not familiar & $20(45.5)$ & $24(54.6)$ & \\
\hline Somewhat familiar & $117(63.6)$ & $67(36.4)$ & \\
\hline Very familiar & $45(48.9)$ & $47(51.1)$ & \\
\hline Likelihood of child to contract HPV & & & .03 \\
\hline Not at all likely & $85(57.8)$ & $62(42.2)$ & \\
\hline Somewhat likely & $93(59.6)$ & $63(40.4)$ & \\
\hline Very likely & $4(25.0)$ & $12(75.0)$ & \\
\hline Important for child to receive HPV vaccine & & & $<.0001$ \\
\hline Not at all important & $42(84.0)$ & $8(16.0)$ & \\
\hline Somewhat important & $96(72.7)$ & $36(27.3)$ & \\
\hline Very important & $43(31.6)$ & $93(68.4)$ & \\
\hline Concerned about side effects of HPV vaccine & & & .56 \\
\hline Not at all concerned & $20(60.6)$ & $13(39.4)$ & \\
\hline Somewhat concerned & $75(53.6)$ & $65(46.4)$ & \\
\hline Very concerned & $86(59.3)$ & $59(40.7)$ & \\
\hline HPV vaccine reduces risk of cervical cancer & & & .001 \\
\hline No & $30(83.3)$ & $6(16.7)$ & \\
\hline Yes & $117(51.5)$ & $110(48.5)$ & \\
\hline No girls & $32(60.4)$ & $21(39.6)$ & \\
\hline HPV vaccine reduces risk of genital warts & & & .06 \\
\hline No & $74(63.8)$ & $42(36.2)$ & \\
\hline Yes & $104(52.8)$ & $93(47.2)$ & \\
\hline Want child to receive HPV vaccine & & & $<.0001$ \\
\hline No & $55(90.2)$ & $6(9.8)$ & \\
\hline Yes & $123(48.2)$ & $132(51.8)$ & \\
\hline Child has received HPV vaccine & & & .008 \\
\hline No & $166(59.7)$ & $112(40.3)$ & \\
\hline Yes & $16(38.1)$ & $26(61.9)$ & \\
\hline Physician supports HPV vaccine & & & .07 \\
\hline No & $4(50.0)$ & $4(50.0)$ & \\
\hline Yes & $43(47.3)$ & $48(52.8)$ & \\
\hline Not Discussed & $135(61.1)$ & $86(38.9)$ & \\
\hline Most influences decision to get HPV vaccine & & & .006 \\
\hline Doctor & $97(51.1)$ & $93(49.0)$ & \\
\hline Spouse & $14(82.4)$ & $3(17.7)$ & \\
\hline Child & $13(48.2)$ & $14(51.9)$ & \\
\hline Parent & $2(28.6)$ & $5(71.4)$ & \\
\hline Mandatory school requirement & $14(70.0)$ & $6(30.0)$ & \\
\hline None & $41(70.7)$ & $17(29.3)$ & \\
\hline Comfortable with mandated HPV vaccine & & & $<.0001$ \\
\hline Not comfortable & $87(97.8)$ & $2(2.3)$ & \\
\hline Somewhat comfortable & $87(53.4)$ & $76(46.7)$ & \\
\hline Very comfortable & $7(10.5)$ & $60(89.6)$ & \\
\hline
\end{tabular}




\begin{tabular}{|c|c|c|c|}
\hline \multirow[b]{2}{*}{ Variable } & \multicolumn{2}{|c|}{$\begin{array}{l}\text { Do You Think the HPV Vaccine } \\
\text { Should be Added to the List of School } \\
\text { Entry Vaccine Requirement? }\end{array}$} & \multirow[b]{2}{*}{$P^{*}$} \\
\hline & No $(\mathrm{n}[\%])$ & Yes (n [\%]) & \\
\hline Mandatory HPV vaccine program helpful & & & $<.0001$ \\
\hline Not helpful & $45(97.8)$ & $1(2.2)$ & \\
\hline Somewhat helpful & $125(64.1)$ & $70(35.9)$ & \\
\hline Very helpful & $12(15.2)$ & $67(84.8)$ & \\
\hline Mandatory HPV vaccine program important & & & $<.0001$ \\
\hline Not important & $47(94.0)$ & $3(6.0)$ & \\
\hline Somewhat important & $123(67.2)$ & $60(32.8)$ & \\
\hline Very important & $12(13.8)$ & $75(86.2)$ & \\
\hline Federal government to pay for HPV vaccine & & & $<.0001$ \\
\hline No & $90(82.6)$ & $19(17.4)$ & \\
\hline Yes & $91(43.3)$ & $119(56.7)$ & \\
\hline Interested in HPV vaccination for child & & & $<.0001$ \\
\hline Not interested & $52(91.2)$ & $5(8.8)$ & \\
\hline Somewhat interested & $100(66.2)$ & $51(33.8)$ & \\
\hline Very interested & $30(26.8)$ & $82(73.2)$ & \\
\hline
\end{tabular}

${ }^{*} \chi^{2}$ test.

HPV, human papillomavirus.

We then examined parents' possible reactions to a mandatory HPV vaccine program: signing a waiver to prevent their child from receiving the HPV vaccine, having their child vaccinated, or doing what their doctor recommends (Table 3). Parents with a child aged 12 to 14 years who were not familiar or very familiar with HPV thought it was very likely their child could contract HPV and thought the HPV vaccine reduced the risk of cervical cancer and genital warts. Those whose physician supported the HPV vaccine more frequently agreed with the statement that they would have their child vaccinated with the HPV vaccine. Subjects who were very comfortable with a mandatory HPV vaccine program thought the federal government should pay for a mandatory HPV vaccine program and were very interested in the HPV vaccine for their child; these parents were more likely to have their child vaccinated.

Several associations with government intervention in HPV vaccination were also examined. Parents who wanted their child to receive the HPV vaccine $(45.5 \%)$ were more likely than those who did not want their child to receive the vaccine $(8.2 \%)$ to want their government representative to make a law requiring the HPV vaccine $(P=.0001)$. Subjects who thought the HPV vaccine should be added to the list of school-entry vaccine require- ments $(84.1 \%)$ were more likely to want their government representative to make a law requiring the HPV vaccine than those who do not think it should be added to the school-entry vaccine requirements (28\%; $P=.0001)$. Individuals with incomes $<\$ 20,000$ were more likely to want the state of Georgia $(P=.004)$ and the federal government $(P=.003)$ to pay for the HPV vaccine for school children than parents at all other income levels (84.4\% and $86.7 \%$, respectively).

\section{Conclusions}

Our study identified numerous factors that increase the likelihood of parents accepting a mandatory HPV vaccination program. Parents with a personal history of HPV-related disease (HPV infection or genital warts) were more likely to support such a mandated program. Parents who had greater familiarity with the adverse consequences of HPV infection were also more apt to approve a school-based mandate. Interestingly, mothers with a history of a previous abnormal Papanicolaou test were not more inclined to support a mandatory program. Many women were unaware of the viral cause for abnormal cervical cytology. A greater number of parents with the lowest income would also comply with a mandatory vaccination program; because 
Table 3. Association of Parental Attitudes, Beliefs, and Possible Responses to a Mandatory Human Papillomavirus Vaccine

\begin{tabular}{|c|c|c|c|c|}
\hline \multirow[b]{2}{*}{ Variable } & \multicolumn{3}{|c|}{$\begin{array}{l}\text { If the HPV Vaccine was Mandatory to Attend School, Which of the } \\
\text { Following Statements Most Agrees with Your View? }\end{array}$} & \multirow[b]{2}{*}{$P^{*}$} \\
\hline & $\begin{array}{c}\text { Would Sign a Waiver to } \\
\text { Prevent my Child from } \\
\text { Receiving the Vaccine } \\
\text { (n [\%]) }\end{array}$ & $\begin{array}{l}\text { Would have My Child } \\
\text { Vaccinated (n [\%]) }\end{array}$ & $\begin{array}{l}\text { Would do Whatever } \\
\text { My Doctor } \\
\text { Recommends } \\
\text { (n [\%]) }\end{array}$ & \\
\hline Gender & & & & .95 \\
\hline Male & $6(16.2)$ & $25(67.6)$ & $6(16.2)$ & \\
\hline Female & $52(18.4)$ & $186(65.7)$ & $45(15.9)$ & \\
\hline Age (years) & & & & .84 \\
\hline $18-29$ & $11(19.6)$ & $36(64.3)$ & $9(16.1)$ & \\
\hline $30-39$ & $23(15.9)$ & $95(65.5)$ & $27(18.6)$ & \\
\hline $40-49$ & $17(18.9)$ & $61(67.8)$ & $12(13.3)$ & \\
\hline $50+$ & $7(24.1)$ & $19(65.5)$ & $3(10.3)$ & \\
\hline Race & & & & .40 \\
\hline White & $40(22.0)$ & $114(62.6)$ & $28(15.4)$ & \\
\hline African American & $18(14.8)$ & $84(68.9)$ & $20(16.4)$ & \\
\hline Asian & $0(0.0)$ & $1(50.0)$ & $1(50.0)$ & \\
\hline Hispanic & $0(0.0)$ & $10(83.3)$ & $2(16.7)$ & \\
\hline Other & $0(0.0)$ & $2(100.0)$ & $0(0.0)$ & \\
\hline Ages of children, 9-11 & & & & .91 \\
\hline No & $25(19.1)$ & $86(65.7)$ & $20(15.3)$ & \\
\hline Yes & $33(17.5)$ & $125(66.1)$ & $31(16.4)$ & \\
\hline Ages of children, $12-14$ & & & & .02 \\
\hline No & $39(18.8)$ & $127(61.4)$ & $41(19.8)$ & \\
\hline Yes & $19(16.8)$ & $84(74.3)$ & $10(8.9)$ & \\
\hline Ages of children, $15-17$ & & & & .23 \\
\hline No & $41(21.0)$ & $123(63.1)$ & $31(15.9)$ & \\
\hline Yes & 17 (13.6) & $88(70.4)$ & $20(16.0)$ & \\
\hline Income & & & & .54 \\
\hline$\leq \$ 20,000$ & $6(13.3)$ & $33(73.3)$ & $6(13.3)$ & \\
\hline$\$ 20,001-\$ 50,000$ & $22(16.5)$ & $88(66.2)$ & $23(17.3)$ & \\
\hline$\$ 50,001-\$ 100,000$ & $21(25.0)$ & $49(58.3)$ & $14(16.7)$ & \\
\hline$>\$ 100,000$ & $9(15.5)$ & $41(70.7)$ & $8(13.8)$ & \\
\hline Education & & & & .84 \\
\hline$>$ High school & $2(15.4)$ & $9(69.2)$ & $2(15.4)$ & \\
\hline High school or GED & $20(16.4)$ & $85(69.7)$ & $17(13.9)$ & \\
\hline$\geq$ College or more & $36(19.5)$ & $117(63.2)$ & $32(17.3)$ & \\
\hline Health insurance & & & & .06 \\
\hline None & $2(11.1)$ & $10(55.6)$ & $6(33.3)$ & \\
\hline Private & $47(21.5)$ & $139(63.5)$ & $33(15.1)$ & \\
\hline Medicaid & $5(7.8)$ & $51(79.7)$ & $8(12.5)$ & \\
\hline Medicare & $0(0.0)$ & $2(100.0)$ & $0(0.0)$ & \\
\hline Other & $3(25.0)$ & $5(41.7)$ & $4(33.3)$ & \\
\hline Tricare & $1(20.0)$ & $4(80.0)$ & $0(0.0)$ & \\
\hline Ever had HPV & & & & .19 \\
\hline Yes & $10(23.8)$ & $30(71.4)$ & $2(4.8)$ & \\
\hline No & $46(17.9)$ & $165(64.2)$ & $46(17.9)$ & \\
\hline Not sure & $2(9.5)$ & $16(76.2)$ & $3(14.3)$ & \\
\hline Ever had genital warts & & & & .22 \\
\hline Yes & $3(20.0)$ & $12(80.0)$ & $0(0.0)$ & \\
\hline No & $55(18.0)$ & $199(65.3)$ & $51(16.7)$ & \\
\hline
\end{tabular}




\begin{tabular}{|c|c|c|c|c|}
\hline \multirow[b]{2}{*}{ Variable } & \multicolumn{3}{|c|}{$\begin{array}{l}\text { If the HPV Vaccine was Mandatory to Attend School, Which of the } \\
\text { Following Statements Most Agrees with Your View? }\end{array}$} & \multirow[b]{2}{*}{$P^{*}$} \\
\hline & $\begin{array}{c}\text { Would Sign a Waiver to } \\
\text { Prevent my Child from } \\
\text { Receiving the Vaccine } \\
\text { (n [\%]) }\end{array}$ & $\begin{array}{l}\text { Would have My Child } \\
\text { Vaccinated (n [\%]) }\end{array}$ & $\begin{array}{l}\text { Would do Whatever } \\
\text { My Doctor } \\
\text { Recommends } \\
\text { (n [\%]) }\end{array}$ & \\
\hline $\begin{array}{l}\text { Abnormal Papanicolaou test (women } \\
\text { only) }\end{array}$ & & & & .62 \\
\hline Yes & $23(19.5)$ & $80(67.8)$ & $15(12.7)$ & \\
\hline No & $31(18.6)$ & $106(63.5)$ & $30(18.0)$ & \\
\hline Not sure & $4(11.4)$ & $25(71.4)$ & $6(17.1)$ & \\
\hline $\begin{array}{l}\text { Support government mandated vaccines } \\
\text { requirements }\end{array}$ & & & & $<.0001$ \\
\hline No & $17(50.0)$ & $10(29.4)$ & $7(20.6)$ & \\
\hline Yes & $40(14.0)$ & $201(70.5)$ & $44(15.4)$ & \\
\hline Familiar with HPV & & & & .02 \\
\hline Not familiar & $7(15.9)$ & $30(68.2)$ & $7(15.9)$ & \\
\hline Somewhat familiar & $30(16.3)$ & $115(62.5)$ & $39(21.2)$ & \\
\hline Very familiar & $21(22.8)$ & $66(71.7)$ & $5(5.4)$ & \\
\hline Likelihood of child to contract HPV & & & & $<.0001$ \\
\hline Not at all likely & $36(24.5)$ & $76(51.7)$ & $35(23.8)$ & \\
\hline Somewhat likely & $20(12.8)$ & $120(76.9)$ & $16(10.3)$ & \\
\hline Very likely & $2(12.5)$ & $14(87.5)$ & $0(0.0)$ & \\
\hline $\begin{array}{l}\text { Important for child to receive HPV } \\
\text { vaccine }\end{array}$ & & & & $<.0001$ \\
\hline Not at all important & $29(58.0)$ & $10(20.0)$ & $11(22.0)$ & \\
\hline Somewhat important & $23(17.4)$ & $81(61.4)$ & $28(21.2)$ & \\
\hline Very important & $5(3.7)$ & $119(87.5)$ & $12(8.8)$ & \\
\hline $\begin{array}{l}\text { Concerned about side effects of HPV } \\
\text { vaccine }\end{array}$ & & & & .08 \\
\hline Not at all concerned & $8(24.2)$ & $20(60.6)$ & $5(15.2)$ & \\
\hline Somewhat concerned & $16(11.4)$ & $102(72.9)$ & $22(15.7)$ & \\
\hline Very concerned & $34(23.5)$ & $87(60.0)$ & $24(16.6)$ & \\
\hline $\begin{array}{l}\text { HPV vaccine reduces risk of cervical } \\
\text { cancer }\end{array}$ & & & & $<.0001$ \\
\hline No & $20(55.6)$ & $10(27.8)$ & $6(16.7)$ & \\
\hline Yes & $25(11.0)$ & $169(74.5)$ & $33(14.5)$ & \\
\hline No girls & $11(20.8)$ & $30(56.6)$ & $12(22.6)$ & \\
\hline $\begin{array}{l}\text { HPV vaccine reduces risk of genital } \\
\text { warts }\end{array}$ & & & & .02 \\
\hline No & $30(25.9)$ & $70(60.3)$ & $16(13.8)$ & \\
\hline Yes & $26(13.2)$ & $136(69.0)$ & $35(17.8)$ & \\
\hline Want child to receive HPV vaccine & & & & $<.0001$ \\
\hline No & $35(57.4)$ & $10(16.4)$ & $16(26.2)$ & \\
\hline Yes & $21(8.24)$ & $199(78.0)$ & $35(13.7)$ & \\
\hline Child has received HPV vaccine & & & & .001 \\
\hline No & $57(20.50)$ & $173(62.2)$ & $48(17.28)$ & \\
\hline Yes & $1(2.4)$ & $38(90.5)$ & $3(7.1)$ & \\
\hline Physician supports HPV vaccine & & & & .0004 \\
\hline No & $3(37.5)$ & $2(25.0)$ & $3(37.5)$ & \\
\hline Yes & $7(7.7)$ & $75(82.4)$ & $9(9.9)$ & \\
\hline Not discussed & $48(21.7)$ & $134(60.6)$ & $39(17.7)$ & \\
\hline
\end{tabular}




\begin{tabular}{|c|c|c|c|c|}
\hline \multirow[b]{2}{*}{ Variable } & \multicolumn{3}{|c|}{$\begin{array}{l}\text { If the HPV Vaccine was Mandatory to Attend School, Which of the } \\
\text { Following Statements Most Agrees with Your View? }\end{array}$} & \multirow[b]{2}{*}{$P^{*}$} \\
\hline & $\begin{array}{l}\text { Would Sign a Waiver to } \\
\text { Prevent my Child from } \\
\text { Receiving the Vaccine } \\
\text { (n [\%]) }\end{array}$ & $\begin{array}{l}\text { Would have My Child } \\
\text { Vaccinated (n [\%]) }\end{array}$ & $\begin{array}{l}\text { Would do Whatever } \\
\text { My Doctor } \\
\text { Recommends } \\
\text { (n [\%]) }\end{array}$ & \\
\hline $\begin{array}{l}\text { Most influences decision to get HPV } \\
\text { vaccine }\end{array}$ & & & & $<.0001$ \\
\hline Doctor & $20(10.5)$ & $137(72.1)$ & $33(17.4)$ & \\
\hline Spouse & $5(29.4)$ & $8(47.1)$ & $4(23.5)$ & \\
\hline Child & $2(7.4)$ & $21(77.8)$ & $4(14.8)$ & \\
\hline Parent & $0(0.0)$ & $5(71.4)$ & $2(28.6)$ & \\
\hline Mandatory school requirement & $4(20.0)$ & $15(75.0)$ & $1(5.0)$ & \\
\hline None & $27(46.6)$ & $24(41.4)$ & $7(12.1)$ & \\
\hline Somewhat comfortable & $8(4.91)$ & $128(78.5)$ & $27(16.6)$ & \\
\hline Very comfortable & $0(0.00)$ & $64(95.5)$ & $3(4.5)$ & \\
\hline $\begin{array}{l}\text { Mandatory HPV vaccine program } \\
\text { helpful }\end{array}$ & & & & $<.0001$ \\
\hline Not helpful & $31(67.4)$ & $9(19.6)$ & $6(13.0)$ & \\
\hline Somewhat helpful & $27(13.9)$ & $127(65.1)$ & $41(21.0)$ & \\
\hline Very helpful & $0(0.0)$ & $75(94.9)$ & $4(5.1)$ & \\
\hline $\begin{array}{l}\text { Mandatory HPV vaccine program } \\
\text { important }\end{array}$ & & & & $<.0001$ \\
\hline Not important & $32(64.0)$ & $12(24.0)$ & $6(12.0)$ & \\
\hline Somewhat important & $25(13.7)$ & $118(64.5)$ & $40(21.9)$ & \\
\hline Very important & $1(1.2)$ & $81(93.1)$ & $5(5.8)$ & \\
\hline $\begin{array}{l}\text { Federal government to pay for HPV } \\
\text { vaccine }\end{array}$ & & & & $<.0001$ \\
\hline No & $42(38.5)$ & $48(44.0)$ & $19(17.4)$ & \\
\hline Yes & $16(7.6)$ & $162(77.1)$ & $32(15.2)$ & \\
\hline Interested in HPV vaccination for child & & & & $<.0001$ \\
\hline Not interested & $41(71.9)$ & $7(12.3)$ & $9(15.8)$ & \\
\hline Somewhat interested & $16(10.6)$ & $102(67.6)$ & $33(21.9)$ & \\
\hline Very interested & $1(0.9)$ & $102(91.1)$ & $9(8.0)$ & \\
\hline $\begin{array}{l}\text { HPV vaccine should be added to the } \\
\text { list of school entry vaccine } \\
\text { requirements }\end{array}$ & & & & $<.0001$ \\
\hline No & $57(31.3)$ & $86(47.3)$ & $39(21.4)$ & \\
\hline Yes & $1(0.7)$ & $125(90.6)$ & $12(8.7)$ & \\
\hline
\end{tabular}

${ }^{*} \chi^{2}$ test.

HPV, human papillomavirus; GED, General Educational Development test.

this vaccine is fairly expensive, one could assume that mandating vaccination might have equaled free availability in their minds. In fact, the Vaccines for Children Program provides free HPV vaccines to indigent children 18 years old or younger. This is a voluntary federal vaccination program that is probably underutilized. The sex, age, race, education, and type of health insurance parents had did not influence their opinion about the importance of a mandated vaccine program. In fact, fewer college- educated parents thought a mandatory HPV vaccine program should be implemented.

Parents who understood the true vulnerability of their child and the possibility of their contracting HPV supported a mandatory HPV vaccine program. Tragically, most parents do not know the ubiquitous nature of $\mathrm{HPV}^{21}$ and awareness of this principle is an important cornerstone for vaccine acceptance. Moreover, no one can advise parents about how their child's immune system might re- 
spond to HPV infection, eg, spontaneous clearance or persistence that may increase the risk for developing a malignancy. Parents who knew that the HPV vaccine reduced the risk of their child developing cervical cancer were more inclined to condone a mandatory program. Further education about HPV and its potential consequences are needed to improve HPV vaccine uptake. Such education could influence response to a mandated program.

Parents whose health care providers supported the HPV vaccine were not more likely to support a mandatory vaccination program. Yet other studies have demonstrated the value of medical advice with respect to HPV vaccine acceptance. ${ }^{19,21,22}$ The opinion of a trusted health care provider is particularly important with respect to preventive medical care. The dialogue between parent and health care provider probably has centered around action (getting the vaccine) and not the particular process (voluntary vs mandatory).

Many other variables indicated that parents thought the HPV vaccine should be added to the list of school-entry vaccine requirements. Parents with no insurance or federally financed insurance more often agreed that the HPV vaccine should be required. Reliance on government-funded heath insurance may have an affect on parental autonomy. These parents may be more willing to abdicate these health decisions to the medical legislative process. However, others caution that such a mandatory program for the HPV vaccine would place a financial burden on existing government health programs, thereby potentially reducing coverage rates for other vaccines. ${ }^{23}$

To parents we posed a realistic scenario: that the HPV vaccine was required for school. They could choose to sign a waiver to prevent their child from receiving the HPV vaccine, have their child vaccinated, or follow the recommendation of their health care provider. Most parents $(66 \%)$ would simply select to allow their child to receive the vaccination. Those parents who supported mandatory vaccination programs knew the potential harms from HPV, realized their child was very susceptible to HPV infection, and wanted their child to receive the vaccine readily accepted compliance with an HPV vaccination mandate. Once again, knowledge about the problem seemed conducive to parents accepting mandatory health pro- grams designed to prevent unnecessary morbidity and mortality.

The remaining parents were fairly divided between the other 2 options. A certain number of parents want to retain the autonomy of making health decisions for their children. In our country and the state in which our study was conducted, opting out of vaccination for religious, medical, or philosophical reasons is allowed. However, some argue that society should protect innocent children who may be hurt by the choices of their parents. ${ }^{24}$ The issue has been conceptualized as conflict between autonomy and utilitarianism. ${ }^{25}$ Other parents place the decision in the hands of their health care provider. Education of these influential people is critically necessary to assure proper guidance is given.

Our study was limited by regional sampling, greater participation by mothers, and limited Asian and Hispanic involvement. A national sample including more fathers and minorities may have altered our findings. Our study was also conducted immediately after unjust negative publicity from the national media. The HPV vaccine controversy is clouded by too many unproven assumptions and unknown factors. ${ }^{26}$ Although acceptance of a mandatory policy for the HPV vaccine may improve in the future, additional research may be beneficial to assess changing and evolving attitudes.

\section{References}

1. Villa LL, Costa RLR, Petta CA, et al. Prophylactic quadrivalent human papillomavirus (types 6, 11, 16 and 18) L1 virus-like particle vaccine in young women: a randomized double-blind placebo-controlled multicentre phase II efficacy trial. Lancet Oncol 2005;6:271-8.

2. FUTURE II Study Group. Quadrivalent vaccine against human papillomavirus to prevent high-grade cervical lesions. N Engl J Med 2007;356:1915-27.

3. Ault KA, FUTURE II Study Group. Effect of prophylactic human papillomavirus L1 virus-like-particle vaccine on risk of cervical intraepithelial neoplasia grade 2, grade 3 and adenocarcinoma in situ: a combined analysis of four randomised clinical trials. Lancet 2007;369:1861-8.

4. Garland SM, Hernandez-Avila M, Wheeler CM, et al. Quadrivalent vaccine against human papillomavirus to prevent anogenital diseases. N Engl J Med 2007;356:1928-43.

5. Associated Press. 1 in 4 U.S. girls received HPV vaccine. October 9, 2008. Available at http:// 
www.msnbc.msn.com/id/27103121/ns/health-kids_ and_parenting/. Accessed 6 July 2009.

6. Springer K. Why are HPV vaccine rates so low? 25 February 2008. Available at http://www.newsweek. com/id/115329/output/print. Accessed 6 July 2009.

7. Markowitz LE, Dunne EF, Saraiya M, Lawson HW, Chesson H, Unger ER. Quadrivalent human papillomavirus vaccine: recommendations of the Advisory Committee on Immunization Practices (ACIP). MMWR Recomm Rep 2007;56:1-24.

8. CDC. Coverage estimates for school entry vaccinations, 2007-2008 school year. Available at http:// www2.cdc.gov/nip/schoolsurv/nationalavg.asp. Accessed 6 July 2009.

9. Ford CA, English A, Davenport AF, Stinnett AJ. Increasing adolescent vaccination: barriers and strategies in the context of policy, legal and financial issues. J Adolesc Health 2009;44:568-74.

10. Kahn JA, Rosenthal SL, Jin Y, Huang B, Namakydoust A, Zimet GD. Rates of human papillomavirus vaccination, attitudes about vaccination, and human papillomavirus prevalence in young women. Obstet Gynecol 2008;111:1103-10.

11. Keating KM, Brewer NT, Gottlieb SL, Liddon N, Ludema C, Smith JS. Potential barriers to HPV vaccine provision among medical practices in an area with high rates of cervical cancer. J Adolesc Health 2008;43:S61-7.

12. Morita JY, Ramirez E, Trick WE. Effect of a schoolentry vaccination requirement on racial and ethnic disparities in hepatitis B immunization coverage levels among public school students. Pediatrics 2008; 121:547-52.

13. Fogarty KJ, Massoudi MS, Gallo W, Averhoff FM, Yusuf H, Fishbein D. Vaccine coverage levels after implementation of a middle school vaccination requirement, Florida, 1997-2000. Public Health Rep 2004;119:163-9.

14. Olshen E, Mahon BE, Wang S, Woods ER. The impact of state policies on vaccine coverage by age 13 in an insured population. J Adolesc Health 2007; 40:405-11.
15. Wilson TR, Fishbein DB, Ellis PA, Edlavitch SA. The impact of a school entry law on adolescent immunization rates. J Adolesc Health 2005;37:511-6.

16. Gostin LO, DeAngelis CD. Mandatory HPV vaccination, public health vs private wealth. JAMA 2007; 297:1921-3.

17. Horn L, Howard C, Waller JL, Ferris DG. Opinions of parent's about school-entry mandates for the human papillomavirus vaccine. J Lower Genit Tract Dis 2010;14:53-8.

18. Hopson K. Majority of U.S. parents not in favor of HPV vaccine mandates. 22 May 2007. Available at http://www2.med.umich.edu/prmc/media/newsroom/ details.cfm?id=532. Accessed 12 January 2010.

19. Ferris DG, Waller JL, Miller J, et al. Variables associated with human papillomavirus (HPV) vaccine acceptance by men. J Am Board Fam Med 2009;22: $34-42$.

20. Ferris DG, Waller JC, Owen A, Smith J. Human papillomavirus vaccine acceptance among mid-adult women. J Am Board Fam Med 2008;21:31-7.

21. Davis K, Dickman ED, Ferris D, Dias JK. Human papillomavirus vaccine acceptability among parents of 10- to 15-year-old adolescents. J Lower Gen Tract Dis 2004;8:188-94.

22. Ferris DG, Waller JL, Miller J, et al. Men's attitudes toward receiving the Human papillomavirus vaccine. J Lower Gen Tract Dis 2008;12:276-81.

23. Javitt G, Berkowitz D, Gostin LO. Assessing mandatory human papillomavirus vaccination: who should call the shots? J Law Med Ethics 2008;36: 384-95.

24. Colgrove J. The ethics and politics of compulsory human papillomavirus vaccination. $\mathrm{N}$ Engl J Med 2006;355:2389-91.

25. Field RI, Caplan AL. A proposed ethical framework for vaccine mandates: Competing values and the case of human papillomavirus. Kennedy Inst Ethics J 2008;18:111-24.

26. Haber G, Malow RM, Zimet GD. The human papillomavirus vaccine mandate controversy. J Pediatr Adolesc Gynecol 2007;20:325-31. 